\title{
How many walking and cycling trips made by elderly are beyond commonly used buffer sizes: Results from a GPS study
}

\author{
R.G. Prins ${ }^{\text {b,* }}$, F. Pierik ${ }^{a}$, A. Etman ${ }^{\text {b }}$, R.P. Sterkenburg ${ }^{a}$, C.B.M. Kamphuis ${ }^{b}$, F.J. van Lenthe ${ }^{b}$ \\ a Department of Urban Environment and Safety, TNO, Utrecht, The Netherlands \\ ${ }^{\mathrm{b}}$ Department of Public Health, Erasmus Medical Center, Rotterdam, The Netherlands
}

\section{A R T I C L E I N F O}

\section{Article history:}

Received 11 September 2013

Received in revised form

23 January 2014

Accepted 29 January 2014

Available online 4 March 2014

Keywords:

Active transport

Buffer

GIS

Distance

Demographic differences

\begin{abstract}
A B S T R A C T
In choosing appropriate buffer sizes to study environmental influences on physical activity, studies are hampered by insufficient insight into the distance elderly travel actively. This study aims at getting insight into the number of trips walked and cycled within various buffer sizes using GPS measures. Data were obtained from the Elderly And their Neighborhood study (Spijkenisse, the Netherlands (2011-2012)). Trip length and mode of transport were derived from the GPS data $(N=120$; total number of trips $=337$ ). Distance decay functions were fitted to estimate the percentage of trips to grocery stores within commonly used buffer sizes. Fifty percent of the trips walked had a distance of at least $729 \mathrm{~m}$; for trips cycled this was $1665 \mathrm{~m}$. Elderly aged under 75 years and those with functional limitations walked and cycled shorter distances than those over 75 years and those without functional limitations. Males cycled shorter distances than females. Distance decay functions may aid the selection of appropriate buffer sizes, which may be tailored to individual characteristics.
\end{abstract}

(c) 2014 Elsevier Ltd. All rights reserved.

\section{Introduction}

In aging societies, health promotion among elderly increasingly focuses on aging in place (Farber et al., 2011; Zantinge et al., 2011). This focus covers both societal need to prevent further increases in costs for institutionalized care, as well as the desire of most elderly people to age in place (Cheek et al., 2005). Physical activity plays an important role in this respect. Being able to walk or cycle to grocery stores contributes substantially to independent living and to health and well-being of elderly (Matthews et al., 2007; Noda et al., 2005; Savela et al., 2010; Smith et al., 2007). It is recognized that physical activity depends on individual characteristics (e.g. age, physical conditions, cognitions towards physical activity) and environmental characteristics (Alfonzo, 2005; Kremers et al., 2006; Sallis et al., 2002) such as the presence of shops (Chaudhury et al., 2012; Van Cauwenberg et al., 2012). Various studies have explored the role of physical environmental factors for walking among elderly. A review of the literature reveals that evidence for such associations is mixed (Van Cauwenberg et al., 2011). These mixed results have been attributed to methodological limitations such as the way buffers to measure environmental characteristics were constructed (i.e. circular vs. road

\footnotetext{
*Corresponding author. Tel.: + 31 107043721; fax: + 31107038474

E-mail address: rick.prins@mrc-epid.cam.ac.uk (R.G. Prins).
}

networks) (Oliver et al., 2007) and their size (i.e. "buffer sizes") (Brownson et al., 2009). Circular buffers become less accurate with the occurrence of physical barriers (e.g. rivers, highways). Therefore, road network buffers are often the preferred methods for constructing a buffer to study environmental correlates of physical activity behavior (Oliver et al., 2007). When choosing an appropriate buffer size to capture environmental characteristics, it is important to know how far people travel actively, which gives an indication of the environmental factors to which people are likely exposed. Auchincloss et al. concluded that there is still little knowledge regarding the relevant buffer size to study the impact of the environment on physical activity (Auchincloss et al., 2012). Some studies have investigated the importance of the size of buffers by studying correlates of physical activity within various buffer sizes (Dowda et al., 2009; Nagel et al., 2008; Prins et al., 2011), but little is known about the number of trips, made by means of active travel, within and beyond these buffers. Information on the relative number of trips made within and beyond these buffers provides insight into the exposure of elderly to their neighborhood environment. We therefore aimed to get insight into the number of trips made beyond various network buffer sizes using GPS measures. This knowledge may aid researchers in constructing appropriately sized network buffers.

A factor that complicates the determination of distances that elderly are willing to travel actively for their grocery shopping is that these distances may differ according to personal factors, such 
as age and gender (Perchoux et al., 2013). This may have consequences for relevant buffer sizes in which environmental influences on active transport behavior are studied. It has been shown that self-reported trip length decreases with increasing age (Yang and Diez-Roux, 2012) and that men seem to cycle less than women (Krizek et al., 2005). In a similar way it is likely that people with functional limitations are more bound to their residential environment and therefore travel shorter distances actively. Insight into how personal characteristics affect the distance people travel actively is needed to come closer towards the individualized buffer sizes where has been called upon (Diez Roux et al., 2007; Perchoux et al., 2013). Therefore, we also examined differences in distances traveled actively according to gender, age, and level of functional limitations.

In summary, it is the aim of this study to a) get insight into the number of trips walked and cycled beyond various buffer sizes using GPS measures and b) examine whether these differ according to gender, age, and level of functional limitations. We hypothesized that, given the high connectivity and relatively good infrastructure for walking and cycling in The Netherlands, most elderly will walk or cycle to get their groceries for distances well beyond $1000 \mathrm{~m}$. In addition, we hypothesized that trip length decreases with increasing age and functional limitations.

\section{Methods}

\subsection{Study design}

This study used cross-sectional data derived from the ELANE (Elderly And their Neighborhood) study, which was conducted in Spijkenisse, a medium-sized city of 73,000 residents (Centraal Bureau voor de Statistiek, 2012) in The Netherlands in 2011-2012. The ELANE study aims at investigating associations between environmental characteristics and physical activity, functional loss, independent living and quality of life in two samples: 1) dismissed hospitalized elderly who participate in the Prevention and Reactivation Care Program (PreCAP) study (de Vos et al., 2012), and 2) randomly selected community-dwelling elderly. In this study we focused on the random sample. The medical ethical committee of the Erasmus Medical Center issued a declaration of no objection for this study.

\subsection{Sampling and procedure}

As part of the ELANE study, a sample of 2017 communitydwelling elderly ( $\geq 65$ years) was randomly drawn from the municipal population registry of Spijkenisse in 2011. Based on their name and address their phone numbers were looked up in online phone number registries. Matched telephone numbers were found for 1190 persons, who received an invitation letter before they were called. In total, 1040 persons answered the phone within 5 attempts. Participants had to be non-institutionalized, not bedridden, community-dwelling, not dependent on a wheelchair and proficient in Dutch. Sixty-eight persons were excluded, as they did not match the above mentioned inclusion criteria. Of the 972 persons eligible for inclusion, 430 were willing to participate (response proportion 44.2\%). Home interviews were carried out by trained research staff between September 2011 and July 2012, excluding the winter months of January to March, in which physical activity levels may be minimal.

The present study used data from a 36\% subsample of the elderly ( $n=156)$ who wore a GPS logger and accelerometer for one week. The subsample consisted of a higher percentage of higher educated individuals than the total sample. No differences were observed in terms of age, gender, weekly minutes spent walking, and weekly minutes spent cycling.

\subsection{Measures}

\subsubsection{Measuring location: GPS receiver}

Elderly in the subsample were instructed to wear a GPS receiver on their right hip (Travel recorder X, BT-Q1000X, QStarz International), from waking hours to bedtime for seven consecutive days. The elderly charged the GPS receiver during the night. A recent study which compared various GPS loggers showed that this GPS logger has a relatively good performance in terms of battery life and accuracy (Duncan et al., 2013). The GPS logger recorded at least every $10 \mathrm{~s}$ the $x$ and $y$ coordinates and time. From these parameters, distance and speed between two points were calculated (Kerr et al., 2012). The GPS data were downloaded by using Q-Travel software and projected as a layer in a GIS database containing street patterns and shops by using the URBIS III software package (TNO, 2012)

\subsubsection{Determination of trips for shopping}

To define the origin of the GPS track, the exact location of the home address was determined by using a center of gravity technique on the GPS signals at night hours (Maas et al., 2013). GPS points were projected in grid cells of 10 by $10 \mathrm{~m}$. Grid cells that contained more than 99 points during night hours were analyzed. In an iterative process, the $x$-and $y$-positions of these grid cells and their 8 surrounding grid cells were averaged to obtain a first estimated location. Subsequently, points at a distance of more than 3 times the root-mean square were considered outliers and were excluded from the analyses. This process was iterated until the new estimated location was at a distance of at most $5 \mathrm{~m}$ from the previous estimated location.

Only trips that started from the home address and ended in the proximity of a grocery store were considered in this study. Data on the locations of grocery stores (i.e. supermarket, bakery, butcher, fish shop, greengrocery) were derived from the "National Information System Labour Organisations" (Landelijk Informatie System Arbeidsorganisaties). All trips that departed from the home address and entered a $30 \mathrm{~m}$ buffer around a grocery store were detected in the data. To be sure that the grocery store was the endpoint of the trip, the GPS signal had to be in the $30 \mathrm{~m}$ buffer for at least $80 \%$ of the time for a period of $2 \mathrm{~min}$. The exact endpoint of a trip was determined by sequentially determining whether a data point $(X)$ was closer to the grocery store than the previous data point $(X-1)$. If this was the case, the data point was part of the trip. When a data point $(X)$ was further than the previous data point $(X-1)$ from the grocery store, the previous data point $(X-1)$ was the endpoint of the trip. The same procedure was applied in the reverse direction towards the home location to find the starting point of the trip.

\subsubsection{Outcome: trip length}

For each trip classified as "grocery shopping", the one-way traveled distance (i.e. the length of the GPS track) between the first point of the track (i.e. the home) and the endpoint of the trip (i.e. the grocery store) was determined. In total 2740 potential trips were automatically identified in the data. Spurious trips were identified and deleted. Trips were considered spurious if all data points were in the cluster that defined the home location. In that case it is likely that the participant was living in the near proximity of a shop. This was the case for one respondent, who was responsible for 1800 "trips". A trip was also considered to be spurious if the average speed was over $150 \mathrm{~km} / \mathrm{h}$, if distances traveled were larger than $5 \mathrm{~km}$, if time traveled was more than 
120 min or less than 2 min. Applying these criteria led to a total number of 337 trips for grocery shopping, made by 120 unique individuals.

\subsubsection{Travel mode}

The mode of travel (i.e. walking or cycling) was determined based on the speed of the GPS logger. The algorithms of Bohte and Maat classify a trip as being walked if the average speed is $<10 \mathrm{~km} / \mathrm{h}$ and the maximum speed is $<14 \mathrm{~km} / \mathrm{h}$, and as being cycled if the average speed is between 10 and $25 \mathrm{~km} / \mathrm{h}$ and the maximum speed is $<45 \mathrm{~km} / \mathrm{h}$ (Bohte and Maat, 2009). Since our sample comprised older adults, we decided to lower these cut-offs. Hence, we categorized a track as being walked if the average speed was less than $6.5 \mathrm{~km} / \mathrm{h}$ and the peak speed was not higher than $9.0 \mathrm{~km} / \mathrm{h}$. A track was defined as cycled if the average speed was between $6.5 \mathrm{~km} / \mathrm{h}$ and $25.0 \mathrm{~km} / \mathrm{h}$ and the peak speed was less than $30.0 \mathrm{~km} / \mathrm{h}$.

\subsubsection{Age, gender and functional limitations}

Age, gender (male: 0/ female: 1) and functional limitations were obtained from the home interview data. Age was categorized in $65-75$ years ( 0 ) and $\geq 75$ years ( 1 ). Functional limitations were assessed according to the criteria of Lawton and Brody (1969). Following the stem item "Do you need help with...", functional limitations were measured with 8 items: 1) using the telephone, 2) traveling, 3) grocery shopping, 4) preparing a meal, 5) household tasks, 6) taking your medicines, 7) finances and 8) doing the laundry. All items had answering categories no (0) and yes (1). These were categorized in not having functional limitations (0) and having at least one functional limitation (1).

\subsection{Statistical analyses}

Descriptive analyses summarized the percentage of trips walked and cycled for shopping purposes, as well as means and medians of distances and time covered.

To mathematically describe the number of trips walked or cycled for utilitarian purposes as a function of distance, so called "distance decay functions" were constructed. For short distances, exponential functions describing distance decay are recommended (Iacono et al., 2010; Signorino et al., 2011):

$P(d)=e^{-\beta d}$ where $P(d)$ is the cumulative percentage of trips with at least distance $d$, and $\beta$ is the distance decay parameter to be estimated. Basically, the decay parameter indicates the steepness of the decline in percentage of trips of at least a given distance. In this function a higher value of the distance decay parameter, $\beta$, means a steeper decline.

To estimate the distance decay parameter, the cumulative percentage of trips was calculated in descending order. Hence, the smallest distance traveled had the highest percentage of trips covering at least that distance (i.e. 100\%), the largest distance had the lowest percentage (approaching 0\%). Subsequently the above mentioned function $\left(e^{-\beta d}\right)$ was fitted on the unique cumulative percentages by using a non-linear ordinary least squares (OLS) regression in Stata:

$n l($ cumpercent $=100 * \exp (-$ decayparam $*$ distance $))$

iterate(10000)vce(clusterid)

In this function, cumpercent was the outcome (i.e. the cumulative percentage of trips with that distance), decayparam was the decay parameter $(\beta)$ to be estimated and distance was the observed distance.

The decay parameter for distance was estimated separately for walking and cycling , for males and females, for those 65-75 and those $75+$ years of age, and for those without and with functional limitations. To visualize the distance decay curves, the functions were plotted together with the observed values. Percentages of trips that fall within a given distance were estimated based on the decay function.

To determine whether two decay parameters were significantly different from each other, an approach suggested by Zou and Donner (2008) was used, to compare two values based on their confidence intervals. Results were considered to be significantly different at $p<0.05$. All analyses were conducted in Stata 12.0.

\section{Results}

\subsection{Characteristics of trips and participants}

In total, 120 unique participants made 337 trips actively for grocery shopping (Table 1 ). Of these 120 persons, $50 \%$ were males, $65.6 \%$ were younger than 75 years and 23\% had at least one functional limitation.

The majority of trips were made by bicycle ( 182 trips vs. 155 by foot) (Table 1). Participants made on average 2.8 trips per week to do groceries, with a range of $1-14$ trips. If participants walked to

Table 1

Characteristics of trips.

\begin{tabular}{|c|c|c|c|}
\hline & Walking & Cycling & Total \\
\hline Number of trips & 155 & 182 & 337 \\
\hline \multicolumn{4}{|l|}{ Number of unique participants } \\
\hline Total & 76 & 94 & 120 \\
\hline Men & 35 & 46 & 60 \\
\hline Women & 41 & 48 & 60 \\
\hline Age $<75$ years & 49 & 64 & 78 \\
\hline Age $\geq 75$ years & 26 & 30 & 42 \\
\hline Without functional limitations & 58 & 80 & 97 \\
\hline With functional limitations & 18 & 14 & 23 \\
\hline \multicolumn{4}{|l|}{ Average number of trips per participant } \\
\hline Total (range) & $2.04(1-7)$ & $1.94(1-11)$ & $2.8(1-14)$ \\
\hline Men (range) & $1.94(1-7)$ & $1.81(1-11)$ & $2.66(1-14)$ \\
\hline Women (range) & $2.12(1-7)$ & $1.88(1-6)$ & $2.95(1-8)$ \\
\hline Age $<75$ years (range) & $1.88(1-7)$ & $1.98(1-11)$ & $2.80(1-14)$ \\
\hline Age $\geq 75$ years (range) & $2.38(1-7)$ & $1.79(1-5)$ & $2.78(1-11)$ \\
\hline Without functional limitations (range) & $1.81(1-7)$ & $1.53(1-11)$ & $2.69(1-14)$ \\
\hline With functional limitations (range) & $2.77(1-7)$ & $1.86(1-6)$ & $3.30(1-13)$ \\
\hline Average trip length (sd) & $1100.6(1130.9)$ & $1997.0(1264.0)$ & $1584.6(1283.4)$ \\
\hline Average trip duration (sd) & $23.2(27.0)$ & $20.3(27.4)$ & $21.6(27.2)$ \\
\hline Average transport time (sd) & $14.7(14.7)$ & $9.8(7.1)$ & $12.1(11.5)$ \\
\hline
\end{tabular}


do groceries, they did this on average 2.0 times per week. If participants cycled to do groceries, they did this on average 1.9 times per week.

In general, more participants cycled at least once, than walking at least once (Table 1). Only among participants with functional limitations, walking was the preferred mode over cycling (i.e. 18 participants with functional limitations walked vs. 14 participants with functional limitations that cycled), which was in contrast to participants without functional limitations of more cycled (80 participants) than walked (58 participants).

The overall average trip length was 1584.6 (SD: 1283.4) m (Table 1). When split out to modality, the trips cycled were on average $1997.0 \mathrm{~m}$ (SD: 1264.0) and the trips walked $1100.6 \mathrm{~m}$ (SD: 1130.9). Average trip duration was $21.6 \mathrm{~min}$ (SD: 27.2) in total, $23.2 \mathrm{~min}$ (SD: 27.0) for walking and $20.3 \mathrm{~min}$ (SD: 27.4) for cycling. The active traveling time was 12.1 (SD: 11.5) min when all trips were considered. The trips cycled had a total active traveling time of $9.8 \mathrm{~min}$ (SD: 7.1), whereas trips walked had an active traveling time of $14.7 \min (\mathrm{SD}: 14.7)$.

\subsection{Trip lengths walked to do groceries}

Table 2 and Fig. 1 describe the estimated percentage of walked trips by distance. With regard to walking, the length of the majority of trips was beyond $500 \mathrm{~m}$ (62\% of trips) (Table 2, Fig. 1). Thirty-nine percent of the trips were estimated to go beyond $1000 \mathrm{~m}, 22 \%$ beyond $1600 \mathrm{~m}$ and $15 \%$ of the trips were beyond $2000 \mathrm{~m}$ (Table 2, Fig. 1). The median trip length was $729 \mathrm{~m}$ (Fig. 1).

Differences in distance decay parameters for age and functional limitations were observed (Table 2). The decay function for those aged 75 years and older was less steep than for those aged 65-74 years, meaning that the trips walked by those aged 75 years and older were overall longer than for those aged 65-74 years. Trips by participants with functional limitations were shorter than those made by participants without functional limitations.

\subsection{Trip lengths cycled to do groceries}

Table 2 and Fig. 1 describe the estimated percentage of cycled trips by distance. The majority (66\%) of the trips cycled were beyond $1000 \mathrm{~m}$ and $51 \%$ beyond $1600 \mathrm{~m}$ (Table 2, Fig. 1). The median trip length was $1665 \mathrm{~m}$ (Fig. 1). Differences in decay parameters were observed for gender, age and functional limitations (Table 2). In general, the distances of trips cycled by men, those aged 75 years and older, and those with functional limitation covered shorter distances than the trips cycled by women, those aged 65-74 years, and those without functional limitation respectively.

\section{Discussion}

The results of this study among elderly in The Netherlands show that a majority of trips to grocery stores were beyond $700 \mathrm{~m}$ when walked and $1600 \mathrm{~m}$ when cycled. The mean trip length was $1101 \mathrm{~m}$ (SD: 1131) for walking and $1997 \mathrm{~m}$ (SD: 1264) for cycling. Walking trips undertaken by 75 years and older, and those without functional limitations, were longer than those undertaken by the elderly aged 65-75 years and those with functional limitation respectively. With regard to cycling, trips made by females, those aged 65-75 years, and those without functional limitations were longer than their counterparts.

An estimated majority of trips using active travel modes to do groceries concerned distances greater than $400 \mathrm{~m}$ (i.e. $68.4 \%$ for trips walked and $84.7 \%$ for trips cycled). This is very similar to the finding by Yang and Diez-Roux (2012) in the general US population that $65 \%$ of the participants traveled distances by foot that were beyond $400 \mathrm{~m}$. Hence, standard conceptualizations of a "walkable" distance of $400 \mathrm{~m}$ (Atash, 1994) may be too short for grocery shopping, since environmental characteristics beyond $400 \mathrm{~m}$ are likely to influence walking and cycling as well. This implies that environmental characteristics beyond $400 \mathrm{~m}$ may influence walking and cycling. Based on the decay parameters presented in this study it is possible to choose a buffer size based on potential exposure. In this lies the challenge of selecting a buffer size that is not too small, but in which the features captured are still reachable. Although still arbitrary, using the median and tertiles of the trip distances at least covered may provide valuable information in selecting a buffer size. We know for instance that $50 \%$ of the trips walked have at least a distance of $729 \mathrm{~m}$; for trips cycled this is $1665 \mathrm{~m}$. Similarly, 33\% of the trips walked had at least a distance of $1165 \mathrm{~m}$ and for the trips cycled this was $2662 \mathrm{~m}$. Hence, buffers between 729 and 1165 m may need to be considered for walking. For cycling these buffers are substantially larger: between 1665 and $2662 \mathrm{~m}$. Indeed, it has been shown that characteristics as far as $1500 \mathrm{~m}$ from a respondent's home address were associated with walking for transport in a general population of adults (McCormack et al., 2008).

Distances traveled by bicycle are longer, which increases the accessibility of facilities. In societies where cycling is a common mode of transport, these trips should (besides walking) be taken into account, as it may greatly enhance the reach of facilities. Similarly, in societies that are more car-reliant, introducing a good cycling infrastructure may enhance accessibility of facilities. Such interventions have recently been undertaken in London (Transport for London, 2008). Hence, in communities where cycling is a common mode of transport, buffers in which environmental characteristics are studied may need to be substantially larger than in communities where cycling is not a common mode of transport.

Like hypothesized, our results suggest that trip length differs according to individual characteristics like gender, age and functional limitations. This may imply that when studying environmental influences on physical activity behaviors, such as active transportation for shopping, one should take these individual characteristics into account in conceptualizing the relevant buffer size (Perchoux et al., 2013). This may be done by defining buffers according to individual characteristics or by statistically weighing the importance of an environmental factor based on the distance from home. The latter may be achieved by using the distancedecay parameters.

We found that men traveled shorter distances by bicycle than women, which confirms findings from Krizek et al. (2005). In addition, our analyses showed that elderly with functional limitations travel shorter distances to grocery stores by active transport than those without functional limitations. It can be argued that those with functional limitations already live near shops (i.e. selective migration) and therefore the distance they have to travel is shorter. However, in additional analyses (not shown; results may be obtained from the first author) we did not find any evidence for this. It is likely that older adults with functional limitations only choose active travel modes if they need to travel a short distance, as their functional limitations and physical complaints impair longer distances. For longer distances it is likely that they choose motorized transport more often.

Interestingly, elderly aged 75 years and over walked longer distances than elderly under 75 years of age. In contrast, they cycle shorter distances. Similarly, those aged 75 years and over walked more frequently than they cycled, whereas the reverse is true for those under 75 years. Other Dutch figures (Dutch Institute for Road Safety Research, 2013) reveal a similar pattern. Compared to cycling (26\% of trips), elderly aged 75 years and over walk more 
Table 2

Distance-decay parameters and estimated percentage of trips walked according to gender, age and functional limitations for commonly used buffer sizes.

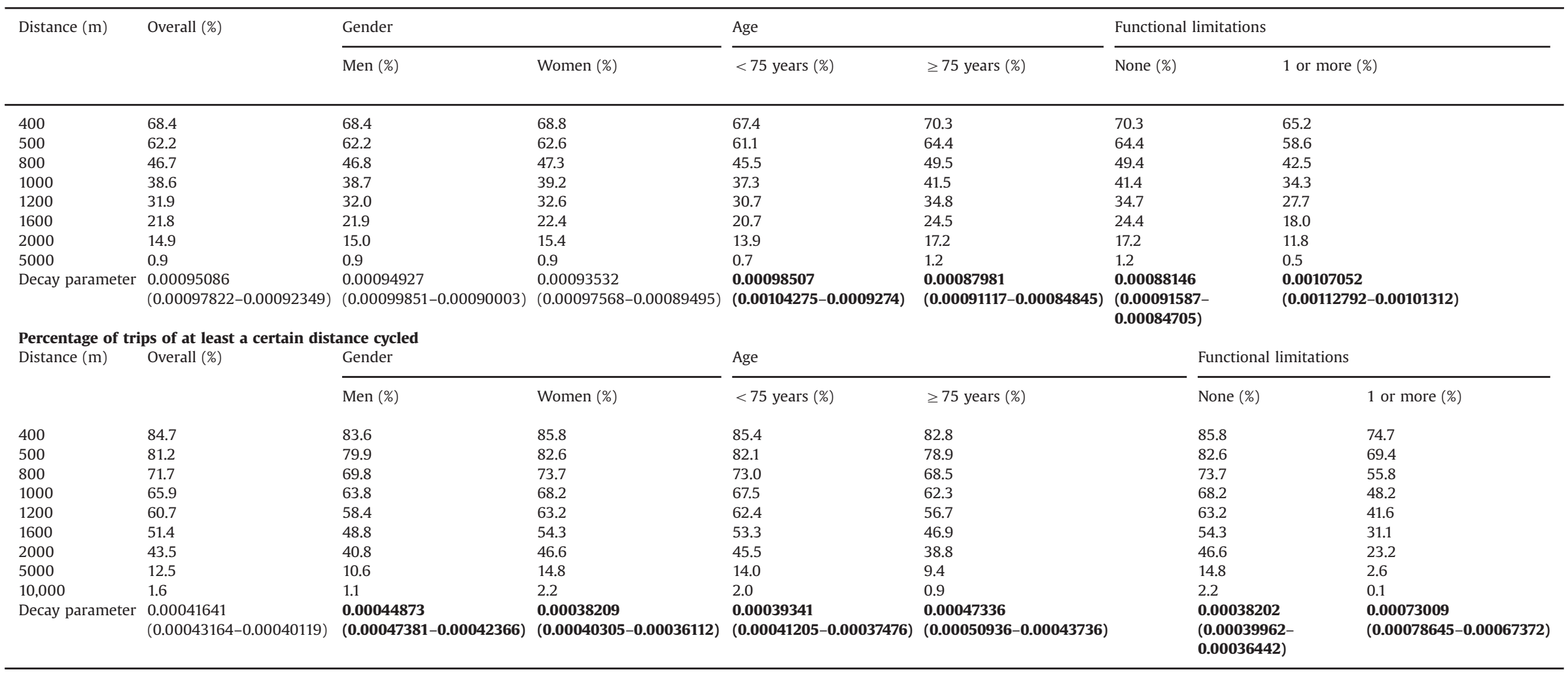

Bold values represent statistically significant differences in decay parameter. 


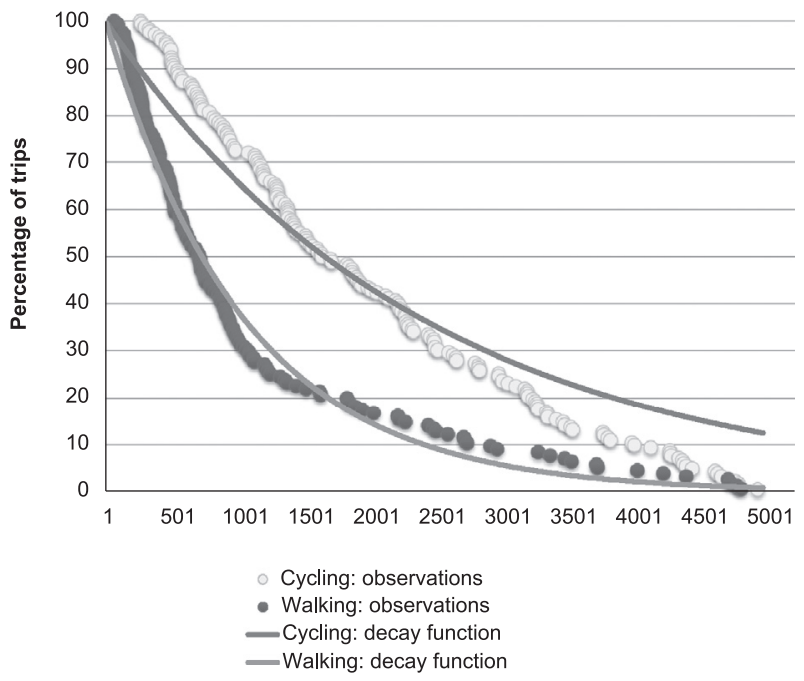

Fig. 1. Fitted distance decay functions on distance data for walking and cycling for shopping in the total study population $(X$-axis=distance in meters, $Y$-axis is cumulative percentage of trips).

frequently (31\% of trips). In contrast, elderly aged $60-74$ years cycled (34\% of trips) more frequently than they walked (20\% of trips) to do their shopping. This may reveal a shift in preference in mode of travel when elderly become older, which may be due to fear of falling, which increases throughout life (Boyd and Stevens, 2009; Jung, 2008). Indeed, fear of falling is an important determinant of quitting cycling among older adults (Anders et al., 2007). In summary, various demographic factors seem to be important in the distance older adults travel actively. A next step would be to explore whether demographic factors interact with each other in their relation to trip length.

This study has several limitations and strengths. A first limitation is the relatively small sample of elderly studied. Moreover we studied only one type of destination (i.e. grocery stores). It has been shown in other research (Yang and Diez-Roux, 2012) that the willingness to travel to a destination differs between types of destination. Therefore, our results are only applicable to active transport to grocery stores, which, however, is one of the most important motives for active transportation (Chaudhury et al., 2012; Michael et al., 2010; Nagel et al., 2008; Rodriguez et al., 2009; Van Cauwenberg et al., 2012). Care should be taken in generalizing the results to other contexts and destinations. The Netherlands is a highly dense country with a unique and highquality walking and cycling infrastructure. However, our results in terms of distances traveled actively are similar to a study conducted in the USA (Yang and Diez-Roux, 2012). Another limitation concerns the classification of travel mode by using GPS loggers; misclassification of travel mode may have occurred (for instance tracks undertaken by using slow driving mobility scooter may have been classified as cycling). Further, we did not adjust for the other demographic factors under study; therefore there may be some confounding present in our analyses. For instance, participants with functional limitations may be older than those without functional limitations-and both age and functional limitations are important to consider when determining trip length. Likewise, the results were not adjusted for seasonal factors. Previous research has shown an influence of seasons on physical activity (Klenk et al., 2012; McMurdo et al., 2012; Tucker and Gilliland, 2007) and active transportation (Yang et al., 2011) For example, the latter study found that active trips were more frequent in the summer months than in the winter months (Yang et al., 2011). Compared to teenagers and children, however, there was relatively little seasonal variability in active travel patterns of adults and elderly (Yang et al., 2011). To minimize seasonal variations in active travel behavior, no measurements took place in the winter months (January, February, March). However we cannot completely rule out seasonal influence on walking behavior. A strength of this study was the use of GPS loggers to objectively determine distances traveled. As we know from self-report that people tend to overestimate their physical activity levels (Prince et al., 2008); using objective measures may overcome this.

To conclude, the use of distance decay functions provided valuable insight into distances traveled by older adults. Most of the trips walked were beyond $700 \mathrm{~m}$ and most of the trips cycled were beyond $1600 \mathrm{~m}$. Trips cycled were considerably longer than trips walked. Even the majority of trips undertaken by a very vulnerable group of elderly with functional limitations were over $500 \mathrm{~m}$ (walked) or $800 \mathrm{~m}$ (cycled). Differences in trip length were observed according to gender, age, and functional limitations. When studying relationships between environmental factors and active transportation, demographic differences as well as common modes of transport should be taken into account. Distance decay functions may be used to aid researchers in selecting appropriate buffer sizes to study environmental influences on physical activity behavior. It further enables the selection of individually tailored buffer sizes.

\section{References}

Alfonzo, M.A., 2005. To walk or not to walk? The hierarchy of walking needs Environ. Behav. 37, 808-836.

Anders, J., Dapp, U., Laub, S., von Renteln-Kruse, W., 2007. Impact of fall risk and fear of falling on mobility of independently living senior citizens transitioning to frailty: screening results concerning fall prevention in the community Z. Gerontol. Geriatr. 40, 255-267.

Atash, F., 1994. Redesigning suburbia for walking and transit: emerging concepts. J. Urban Plan. Dev. 120, 48-57.

Auchincloss, A.H., Gebreab, S.Y., Mair, C., Diez Roux, A.V., 2012. A review of spatial methods in epidemiology, 2000-2010. Annu. Rev. Public Health 33, 107-122.

Bohte, W., Maat, K., 2009. Deriving and validating trip purposes and travel modes for multi-day GPS-based travel surveys: a large-scale application in The Netherlands. Transp. Res. Part C, 17.

Boyd, R., Stevens, J.A., 2009. Falls and fear of falling: burden, beliefs and behaviors Age Ageing 38, 423-428.

Brownson, R.C., Hoehner, C.M., Day, K., Forsyth, A., Sallis, J.F., 2009. Measuring the built environment for physical activity: state of the science. Am. J. Prev. Med. 36, S99-123 S99S123.

Centraal Bureau voor de Statistiek, StatLine, 2012.

Chaudhury, H., Mahmood, A., Michael, Y., Campo, M., Hay, K., 2012. The influence of neighborhood residential density, physical and social environments on older adults' physical activity: an exploratory study in two metropolitan areas. J. Aging Stud. 26, 35-43.

Cheek, P., Nikpour, L., Nowlin, H.D., 2005. Aging well with smart technology. Nurs Adm. Q. 29, 329-338.

de Vos, A.J., Asmus-Szepesi, K.J., Bakker, T.J., de Vreede, P.L., van Wijngaarden, J.D. Steyerberg, E.W., Mackenbach, J.P., Nieboer, A.P., 2012. Integrated approach to prevent functional decline in hospitalized elderly: the Prevention and Reactivation Care Program (PReCaP). BMC Geriatr. 12 (7).

Diez Roux, A.V., Evenson, K.R., McGinn, A.P., Brown, D.G., Moore, L., Brines, S., Jacobs D.R., 2007. Availability of recreational resources and physical activity in adults. Am. J. Public Health 97, 493-499.

Dowda, M., Dishman, R.K., Porter, D., Saunders, R.P., Pate, R.R., 2009. Commercial facilities, social cognitive variables, and physical activity of 12th grade girls. Ann. Behav. Med. 37, 77-87.

Duncan, S., Stewart, T.I., Oliver, M., Mavoa, S., Macrae, D., Badland, H.M., Duncan, M.J., 2013. Portable global positioning system receivers: static validity and environmental conditions. Am. J. Prev Med. 44, e19-e29.

Dutch Institute for Road Safety Research, National travel survey, 2013.

Farber, N., Shinkle, D., Lynott, L., Fox-Grage, W., \& Harrell, R. (2011). Aging in place: A state survey of livability policies and practices. Retrieved June 14, 2013, from http://assets.aarp.org/rgcenter/ppi/liv-com/aging-in-place-2011-full.pdf.

Iacono, M., Krizek, K., El-Geneidy, A., 2010. Measuring non-motorized accessibility issues, alternatives, and execution. J. Transp. Geogr. 18, 133-140.

Jung, D., 2008. Fear of falling in older adults: comprehensive review. Asian Nurs. Res. 2, 214-222.

Kerr, J., Marshall, S., Godbole, S., Neukam, S., Crist, K., Wasilenko, K., Golshan, S. Buchner, D., 2012. The relationship between outdoor activity and health in older adults using GPS. Int. J. Environ. Res. Public Health 9, 4615-4625. 
Klenk, J., Buchele, G., Rapp, K., Franke, S., Peter, R., 2012. Walking on sunshine: effect of weather conditions on physical activity in older people. J. Epidemiol. Community Health 66, 474-476.

Kremers, S.P., de Bruijn, G.J., Visscher, T.L., van Mechelen, W., de Vries, N.K., Brug, J., 2006. Environmental influences on energy balance-related behaviors: a dualprocess view. Int. J. Behav. Nutr. Phys. Act. 3, 9.

Krizek, K.J., Johnson, P.J., Tilahun, N., 2005. Gender differences in bicycling behavior and facility preferences. Res. Women's Issues Transp. 2, 31-40.

Lawton, M.P., Brody, E.M., 1969. Assessment of older people: self-maintaining and instrumental activities of daily living. Gerontologist 9, 179-186.

Maas, J., Sterkenburg, R.P., de Vries, S.I., Pierik, F., 2013. Using GPS to Measure the Interaction Between Individuals and Their Neighborhood, Neighborhood Structure and Health Promotion. Springer, New York, pp. 153-175.

Matthews, C.E., Jurj, A.L., Shu, X.O., Li, H.L., Yang, G., Li, Q., Gao, Y.T., Zheng, W., 2007 Influence of exercise, walking, cycling, and overall nonexercise physical activity on mortality in Chinese women. Am. J. Epidemiol. 165, 1343-1350.

McCormack, G.R., Giles-Corti, B., Bulsara, M., 2008. The relationship between destination proximity, destination mix and physical activity behaviors. Prev. Med. 46, 33-40.

McMurdo, M.E.T., Argo, I., Crombie, LK. Feng, Z, Sniehotta, FF, Vadiveloo, T, Witham, M.D., Donnan, P.T., 2012. Social, environmental and psychological factors associated with objective physical activity levels in the over 65 s. PLoS One 7, e31878.

Michael, Y.L., Perdue, L.A., Orwoll, E.S., Stefanick, M.L., Marshall, L.M., 2010. Physica activity resources and changes in walking in a cohort of older men. Am. J. Public Health 100, 654-660.

Nagel, C.L., Carlson, N.E., Bosworth, M., Michael, Y.L., 2008. The relation between neighborhood built environment and walking activity among older adults. Am. J. Epidemiol. 168, 461-468.

Noda, H., Iso, H., Toyoshima, H., Date, C., Yamamoto, A., Kikuchi, S., Koizumi, A. Kondo, T., Watanabe, Y., Wada, Y., Inaba, Y., Tamakoshi, A., 2005. Walking and sports participation and mortality from coronary heart disease and stroke. J. Am. Coll. Cardiol. 46, 1761-1767.

Oliver, L.N., Schuurman, N., Hall, A.W., 2007. Comparing circular and network buffers to examine the influence of land use on walking for leisure and errands. Int. J. Health Geogr. 6, 41.

Perchoux, C., Chaix, B., Cummins, S., Kestens, Y., 2013. Conceptualization and measurement of environmental exposure in epidemiology: accounting for activity space related to daily mobility. Health Place 21, 86-93.

Prince, S.A., Adamo, K.B., Hamel, M.E., Hardt, J., Connor Gorber, S., Tremblay, M. 2008. A comparison of direct versus self-report measures for assessing physical activity in adults: a systematic review. Int. J. Behav. Nutr. Phys. Act. 5, 56.
Prins, R.G., Ball, K., Timperio, A., Salmon, J., Oenema, A., Brug, J., Crawford, D., 2011. Associations between availability of facilities within three different neighborhood buffer sizes and objectively assessed physical activity in adolescents. Health Place $17,1228-1234$.

Rodriguez, D.A., Evenson, K.R., Diez Roux, A.V., Brines, S.J., 2009. Land use, residential density, and walking. The multi-ethnic study of atherosclerosis. Am. J. Prev. Med. 37, 397-404.

Sallis, J.F., Kraft, K., Linton, L.S., 2002. How the environment shapes physical activity: a transdisciplinary research agenda. Am. J. Prev. Med. 22, 208.

Savela, S.L., Koistinen, P., Tilvis, R.S., Strandberg, A.Y., Pitkälä, K.H., Salomaa, V.V., Miettinen, T.A., Strandberg, T.E., 2010. Physical activity at midlife and healthrelated quality of life in older men. Arch. Intern. Med. 170, 1171-1172.

Signorino, G., Pasetto, R., Gatto, E., Mucciardi, M., La Rocca, M., Mudu, P., 2011. Gravity models to classify commuting vs. resident workers. An application to the analysis of residential risk in a contaminated area. Int. J. Health Geogr. 10, 11.

Smith, T.C., Wingard, D.L., Smith, B., Kritz-Silverstein, D., Barrett-Connor, E., 2007. Walking decreased risk of cardiovascular disease mortality in older adults with diabetes. J. Clin. Epidemiol. 60, 309-317.

Transport for London, Central London Congestion Charging: Impacts monitoring, 2008

Tucker, P., Gilliland, J., 2007. The effect of season and weather on physical activity: a systematic review. Public Health 121, 909-922.

Van Cauwenberg, J., De Bourdeaudhuij, I., De Meester, F., Van Dyck, D., Salmon, J., Clarys, P., Deforche, B., 2011. Relationship between the physical environment and physical activity in older adults: a systematic review. Health Place 17, 458-469.

Van Cauwenberg, J., Van Holle, V., Simons, D., Deridder, R., Clarys, P., Goubert, L. Nasar, J., Salmon, J., De Bourdeaudhuij, I., Deforche, B., 2012. Environmental factors influencing older adults' walking for transportation: a study using walkalong interviews. Int. J.Behav. Nutr. Phys. Act. 9, 85.

Yang, Y., Diez-Roux, A.V., 2012. Walking distance by trip purpose and population subgroups. Am. J. Prev. Med. 43, 11-19.

Yang, Y., Diez Roux, A.V., Bingham, C.R., 2011. Variability and seasonality of active transportation in USA: evidence from the 2001 NHTS. Int. J. Behav. Nutr. Phys. Act. 8, 96.

Zantinge, E.M., van der Wilk, E.A., van Wieren, S., Schoemaker, C.G., 2011. Gezond ouder worden in Nederland [Healthy ageing in The Netherlands]National Institute for Public Health and the Environment, Bilthoven.

Zou, G.Y., Donner, A., 2008. Construction of confidence limits about effect measures: a general approach. Stat. Med. 27, 1693-1702. 